\title{
Broadband microwave spectroscopy of semiconductor nanowire-based Cooper-pair
} transistors

Proutski, Alex; Laroche, Dominique; van 't Hooft, Bas; Krogstrup, Peter; Nygard, Jesper;

Kouwenhoven, Leo P.; Geresdi, Attila

\section{Published in:}

Physical Review B

DOI:

10.1103/PhysRevB.99.220504

Publication date:

2019

Document version

Publisher's PDF, also known as Version of record

Citation for published version (APA):

Proutski, A., Laroche, D., van 't Hooft, B., Krogstrup, P., Nygard, J., Kouwenhoven, L. P., \& Geresdi, A. (2019).

Broadband microwave spectroscopy of semiconductor nanowire-based Cooper-pair transistors. Physical Review B, 99(22), [220504]. https://doi.org/10.1103/PhysRevB.99.220504 


\title{
Broadband microwave spectroscopy of semiconductor nanowire-based Cooper-pair transistors
}

\author{
Alex Proutski, ${ }^{1,{ }^{*}}$ Dominique Laroche,,${ }^{1, *}$ Bas van 't Hooft, ${ }^{1}$ Peter Krogstrup, ${ }^{2,3}$ Jesper Nygård, ${ }^{3}$ \\ Leo P. Kouwenhoven, ${ }^{4,1}$ and Attila Geresdi ${ }^{1, \dagger}$ \\ ${ }^{1}$ QuTech and Kavli Institute of Nanoscience, Delft University of Technology, 2600 GA Delft, The Netherlands \\ ${ }^{2}$ Microsoft Quantum Materials Lab Copenhagen, Niels Bohr Institute, University of Copenhagen, 2100 Copenhagen, Denmark \\ ${ }^{3}$ Center for Quantum Devices, Niels Bohr Institute, University of Copenhagen, 2100 Copenhagen, Denmark \\ ${ }^{4}$ Microsoft Quantum Lab Delft, 2600 GA Delft, The Netherlands
}

(Received 31 January 2019; revised manuscript received 10 May 2019; published 10 June 2019)

\begin{abstract}
The Cooper-pair transistor (CPT), a small superconducting island enclosed between two Josephson weak links, is the atomic building block of various superconducting quantum circuits. Utilizing gate-tunable semiconductor channels as weak links, the energy scale associated with the Josephson tunneling can be changed with respect to the charging energy of the island, tuning the extent of its charge fluctuations. Here, we directly demonstrate this control by mapping the energy level structure of a CPT made of an indium arsenide nanowire with a superconducting aluminum shell. We extract the device parameters based on the exhaustive modeling of the quantum dynamics of the phase-biased nanowire CPT and directly measure the even-odd parity occupation ratio as a function of the device temperature, relevant for superconducting and prospective topological qubits.
\end{abstract}

DOI: 10.1103/PhysRevB.99.220504

The energy landscape of a Cooper-pair transistor (CPT), a mesoscopic superconducting island coupled to superconducting leads via two Josephson junctions, is determined by the interplay of the electrostatic addition energy of a single Cooper pair, $E_{C}=(2 e)^{2} / 2 C[1,2]$, and the coherent tunneling of Cooper pairs, characterized by the Josephson energy $E_{J}[3,4]$.

The electronic transport through CPTs has mostly been studied for metallic superconducting islands enclosed between tunnel junctions by voltage bias spectroscopy [5-7], switching current measurements [8-11], microwave reflectometry $[12,13]$, and broadband microwave spectroscopy $[14,15]$. Recent material developments $[16,17]$ made it possible to investigate superconducting transport in semiconductor nanowire (NW) weak links, which lead to Andreev level quantum circuits [18-20] and gate-tunable superconducting quantum devices [21-24]. In addition, hybrid superconductor-semiconductor island devices, which are the atomic building blocks of proposed topological quantum bits based on Majorana zero-energy modes [25-28], have been fabricated and measured using normal metallic leads [29,30], but thus far there is very limited experimental work on hybrid CPTs with superconducting leads [31].

Such applications require the control of the Josephson coupling via the semiconductor weak link [32]. In addition, the charging energy of a NW CPT can deviate from the predictions of the orthodox theory $[1,2]$ due to renormalization effects arising because of finite channel transmissions [33]. Therefore, understanding the quantum dynamics of CPTs with semiconductor weak links is crucial for these hybrid device architectures.

\footnotetext{
*These authors contributed equally to this work.

${ }^{\dagger}$ Corresponding author: a.geresdi@tudelft.nl
}

Here, we directly measure the transitions between the energy levels of a NW CPT. The CPT is embedded in the circuit shown in Fig. 1(a). The superconducting island is created from an indium arsenide (InAs) nanowire with an epitaxial layer of aluminum (Al) [16] between two Josephson junctions, formed by removing two sections of the Al shell with a wet chemical etch. We investigated two devices, both with 100-nm-long junctions and island lengths of $800 \mathrm{~nm}$ and $1.75 \mu \mathrm{m}$ for device 1 and device 2 [enclosed in the red box in Fig. 1(a)], respectively. The junctions are tuned via their respective local electrostatic gates $V_{\mathrm{tg} 1}$ and $V_{\mathrm{tg} 2}$. The gate charge, $n_{\mathrm{g}}=V_{\mathrm{g}} C_{\mathrm{g}} / 2 e$, is set by the gate voltage $V_{\mathrm{g}}$ and the effective gate capacitance $C_{\mathrm{g}}$ [see the right panel in Fig. 1(a) and the Supplemental Material [34]]. The nanowire CPT is embedded in a superconducting quantum interference device (SQUID) with an $\mathrm{Al} / \mathrm{AlO}_{x} / \mathrm{Al}$ tunnel junction [in the yellow box in Fig. 1(a)] which exhibits a much higher Josephson energy than the CPT. This asymmetry ensures that the applied phase $\varphi=2 \pi \Phi / \Phi_{0}$ drops mostly over the CPT. Here, $\Phi$ is the applied flux and $\Phi_{0}=h / 2 e$ is the superconducting flux quantum.

We utilized a capacitively coupled $\mathrm{Al} / \mathrm{AlO}_{x} / \mathrm{Al}$ superconducting tunnel junction as a broadband on-chip microwave spectrometer [green box in Fig. 1(b)] [14,18,35], where inelastic Cooper-pair tunneling gives rise to a dc current contribution in a dissipative environment [36],

$$
I_{\text {spec }}=\frac{I_{\mathrm{c}, \mathrm{spec}}^{2} \operatorname{Re}[Z(\omega)]}{2 V_{\mathrm{spec}}} .
$$

Here, $I_{\mathrm{c}, \text { spec }}$ is the critical current of the spectrometer tunnel junction and $Z(\omega)$ is the impedance of the environment at the frequency $\omega=2 e V_{\text {spec }} / \hbar$, determined by the spectrometer $\mathrm{dc}$ voltage bias $V_{\text {spec }}$ [Fig. 1(d)]. This de to microwave conversion allowed us to directly measure the excitation energies of 
(a)
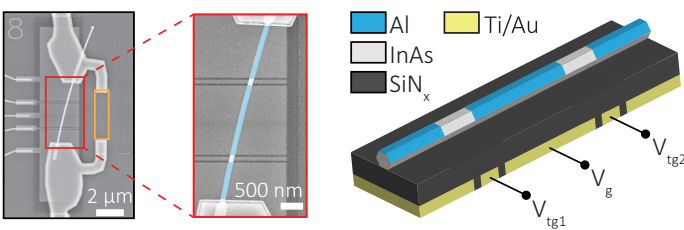

(b)

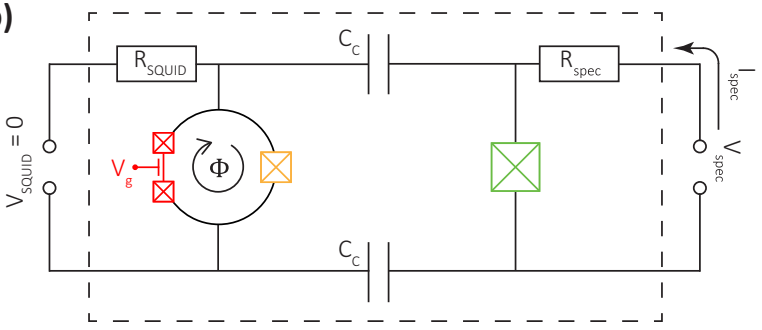

(c)
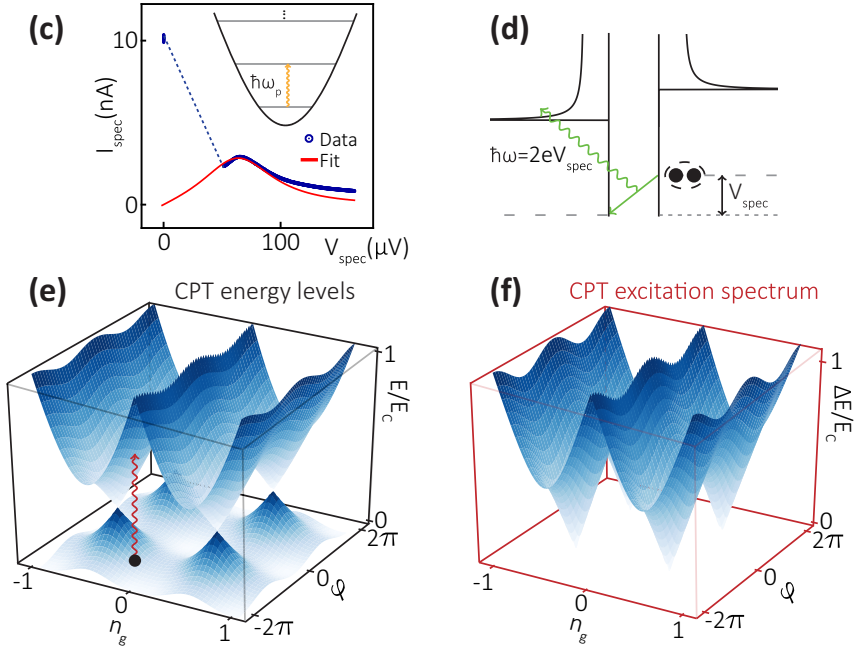

FIG. 1. (a) Left: Scanning electron micrograph of the nanowire $\mathrm{CPT}$ (in red box) and an $\mathrm{Al} / \mathrm{AlO}_{x} / \mathrm{Al}$ tunnel junction (yellow box) forming the hybrid SQUID loop. Middle: False colored micrograph of the nanowire CPT (device 2). Right: Three-dimensional sketch of the CPT on the three electrostatic gates. (b) Equivalent circuit schematics with the hybrid SQUID on the left and a single $\mathrm{Al} / \mathrm{AlO}_{x} / \mathrm{Al}$ tunnel junction used as a spectrometer (green box) on the right side. The circuit elements within the black dashed box are on chip and cooled to $T \sim 18 \mathrm{mK}$. (c) $I(V)$ trace of the spectrometer with the CPT arm in full depletion (device 1). The red solid line shows the fit to the circuit model of a single resonance centered at $\hbar \omega_{p}=148 \mu \mathrm{eV}$ driven by the photons with an energy of $\hbar \omega=$ $2 e V_{\text {spec }}$ emitted by the spectrometer junction (d). The calculated energy bands (e) and transition energies (f) of a CPT with $E_{J 1}=$ $E_{J 2}=E_{C} / 4$ as a function of gate charge $n_{\mathrm{g}}$ and total phase bias $\varphi$.

the hybrid SQUID, where $\operatorname{Re}[Z(\omega)]$ exhibits a local maximum [37]. To reduce microwave leakage, we applied the bias voltages to the hybrid SQUID and to the spectrometer junctions via on-chip resistors, yielding $R_{\text {SQUID }}=12 \mathrm{k} \Omega$ and $R_{\mathrm{spec}}=2.8 \mathrm{k} \Omega$. The chip [in the black dashed box in Fig. 1(b)] was thermally anchored to the mixing chamber of the dilution refrigerator with a base temperature of $\approx 18 \mathrm{mK}$. Full details of the fabrication process and device geometry are given in the Supplemental Material [34].

We begin by analyzing the circuit while keeping both nanowire junctions in full depletion by applying large negative gate voltages $V_{\mathrm{tg} 1}$ and $V_{\mathrm{tg} 2}$. The $I(V)$ curve of the spectrometer (a)

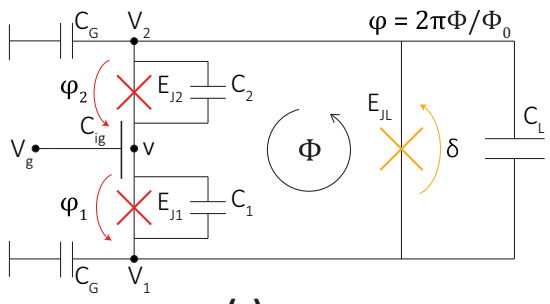

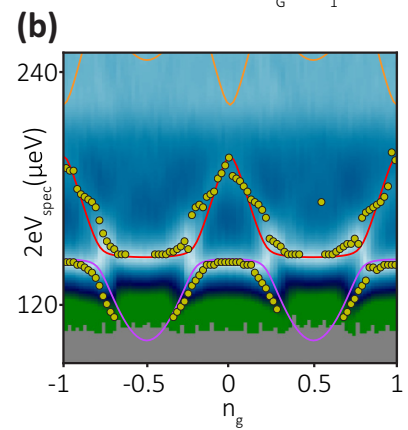

(d)

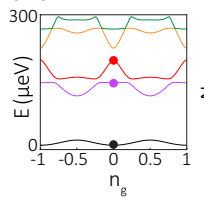

(e)

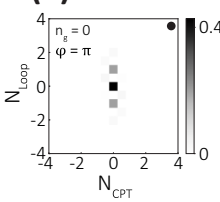

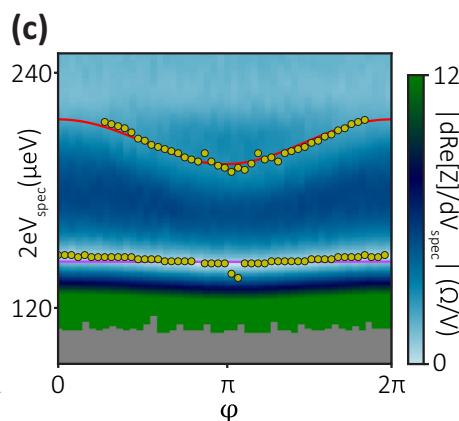

(f)

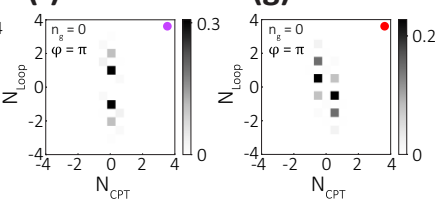

FIG. 2. (a) Equivalent schematics of the hybrid SQUID used to build the circuit Hamiltonian. Observed transitions for device 1 as a function of the gate charge $n_{\mathrm{g}}$ at a fixed $\varphi=\pi$ (b) and applied phase bias $\varphi=2 \pi \Phi / \Phi_{0}$ at a fixed $n_{\mathrm{g}}=0(\mathrm{c})$. The transitions are identified at the local minima of $\left|d \operatorname{Re}(Z) / d V_{\text {spec }}\right|$ (yellow dots). The best fit is shown as solid lines, yielding $E_{C 1}=168 \mu \mathrm{eV}, E_{C 2}=$ $260 \mu \mathrm{eV}, E_{C c}=188 \mu \mathrm{eV}, E_{J 1}=132 \mu \mathrm{eV}$, and $E_{J 2}=16 \mu \mathrm{eV}$ (see text). (d) The corresponding energy bands of the device as a function of $n_{\mathrm{g}}$ at $\varphi=\pi$. The two-component probability distributions of the ground state (e), first excited state (f), and second excited state (g) at $n_{\mathrm{g}}=0$ and $\varphi=\pi$, denoted by circles of the corresponding color in (d) (see text). See Fig. 3 upper row for gate voltage values.

of device 1 is shown in Fig. 1(c). A clear peak is observed with an amplitude of $3 \mathrm{nA}$ centered at $\approx 75 \mu \mathrm{eV}$. We attribute this peak to the plasma resonance of the tunnel junction in the SQUID at $\hbar \omega_{p}=\sqrt{2 E_{J L} E_{C L}}$. Here, $E_{J L}=\Delta_{J} h /\left(8 e^{2} R_{J}\right)=$ $249 \mu \mathrm{eV}$ is the Josephson energy [4], with $\Delta_{J}=245 \mu \mathrm{eV}$ being the measured superconducting gap and $R_{J}=3.17 \mathrm{k} \Omega$ the normal state resistance of the junction, acquired at a voltage bias much higher than $2 \Delta_{J}$. This value yields $E_{C L}=$ $2 e^{2} / C_{L}=44 \mu \mathrm{eV}$ and a shunt capacitance $C_{L}=7.28 \mathrm{fF}$. Fitting the resonant peak using Eq. (1), we find a quality factor $Q \approx 1$ and a characteristic impedance $Z_{0}=610 \Omega \ll$ $R_{q}=h / 4 e^{2}$, which together ensure the validity of Eq. (1) describing a direct correspondence between the measured $I_{\text {spec }}$ and $\operatorname{Re}[Z(\omega)]$. We note that we found very similar values for device 2 as well (see Supplemental Material for a detailed analysis and a list of parameters [34]).

Next, we investigate the spectrometer response to the applied gate voltage $V_{\mathrm{g}}$ and phase $\varphi$ [Figs. 2(b) and 2(c)] when the Josephson junctions are opened by setting positive gate voltages $V_{\mathrm{tg} 1}$ and $V_{\mathrm{tg} 2}$. The excitations of the CPT are superimposed on that of the plasma resonance, so we display $\left|d \operatorname{Re}(Z) / d V_{\text {spec }}\right|$ to reach a better visibility of the transitions 
(see the Supplemental Material for a comparison [34]). Note that we show the excitation energy $\hbar \omega=2 e V_{\text {spec }}$ on the vertical axis for all spectra. This measurement yields clear oscillations as a function of both $n_{\mathrm{g}}$ and $\varphi$, consistent with the expected periodic behavior of the CPT energy levels [8]. We note that the finite load resistance of the spectrometer $R_{\text {spec }}$ prevented us from measuring the transitions below $2 \mathrm{eV}$ spec $=$ $103 \mu \mathrm{eV}$.

We model our device with the schematics depicted in Fig. 2(a) and build the Hamiltonian of the circuit based on conventional quantization procedures [38,39]. We use the conjugate charge and phase operators which pairwise obey $\left[\hat{\varphi}_{1,2}, \hat{N}_{1,2}\right]=i$ and note that $\hat{\delta}=\varphi-\hat{\varphi}_{1}-\hat{\varphi}_{2}$,

$$
\begin{aligned}
\hat{H}= & \frac{1}{2} E_{C 1}\left(\hat{N}_{1}-n_{\mathrm{g}}\right)^{2}+\frac{1}{2} E_{C 2}\left(\hat{N}_{2}+n_{\mathrm{g}}\right)^{2} \\
& -\frac{1}{2} E_{C c}\left(\hat{N}_{1}-n_{\mathrm{g}}\right)\left(\hat{N}_{2}+n_{\mathrm{g}}\right) \\
& -E_{J 1} \cos \left(\hat{\varphi}_{1}\right)-E_{J 2} \cos \left(\hat{\varphi}_{2}\right) \\
& -E_{J L} \cos \left(\varphi-\hat{\varphi}_{1}-\hat{\varphi}_{2}\right) .
\end{aligned}
$$

Here, the charging of the circuit is described by the effective parameters $E_{C 1}, E_{C 2}$, and $E_{C c}$ set by the capacitance values $C_{1}, C_{2}, C_{L}, C_{i g}$, and $C_{G}$ with a functional form provided in the Supplemental Material [34]. The Cooper-pair tunneling is characterized by the Josephson energies of the three junctions $E_{J 1}, E_{J 2}$ and $E_{J L}$, respectively. We note that we set $E_{J L}=$ $249 \mu \mathrm{eV}$ for the analysis below.

To calculate the excitation spectrum, we solve the eigenvalue problem to find $E_{i}\left(n_{\mathrm{g}}, \varphi\right)$, where $\hat{H} \Psi_{i}=E_{i} \Psi_{i}$, and compute the transition energies $\hbar \omega_{i}=E_{i}-E_{0}$, with $E_{0}$ being the ground-state energy of the system. This model allows us to fit the excitation spectra simultaneously as a function of $n_{\mathrm{g}}$ and $\varphi$ based on the first two transitions (red and purple solid lines for $\hbar \omega_{1}$ and $\hbar \omega_{2}$, respectively) against the measured data (yellow circles in Fig. 2). For illustration, we also display $\hbar \omega_{3}$ (orange line) in Fig. 2(b) using the same fit parameters, however, this transition was not observed in the experiment.

To understand the nature of the excited levels, we calculate the energy bands of the hybrid SQUID using the fitted parameters [Fig. 2(d)] and evaluate the probability distribution $p_{i}\left(N_{1}, N_{2}\right)=\left|\Psi_{i}\left(N_{1}, N_{2}\right)\right|^{2}$, where $N_{1}$ and $N_{2}$ form the charge computational basis. However, it is more instructive to use the charge numbers $N_{\text {CPT }}=N_{1}-N_{2}$ and $N_{\text {Loop }}=N_{1}+N_{2}$. Intuitively, $N_{\text {CPT }}$ and $N_{\text {Loop }}$ represent the excess number of Cooper pairs on the island and in the loop, respectively. Indeed, the ground-state wave function is centered around $N_{\text {CPT }}=N_{\text {Loop }}=0$ [Fig. 2(e)]. Conversely, the probability distribution of the first excited state [Fig. 2(f)] exhibits a bimodal distribution in $N_{\text {Loop }}$, consistently with the first plasma mode excitation but no excess charge on the CPT [purple circle in Fig. 2(d)]. This is in contrast with the wave function of the next energy level [Fig. 2(g) and the red circle in Fig. 2(d)], which is centered around $N_{\mathrm{CPT}}= \pm 1$. This analysis demonstrates the coupling between the plasma and localized charge degrees of freedom [40].

Next, we investigate the impact of $V_{\operatorname{tg} 1}$ and $V_{\operatorname{tg} 2}$ on the CPT spectrum. In Fig. 3, we show the measured spectra for two distinct gate settings. Remarkably, almost a full suppression of
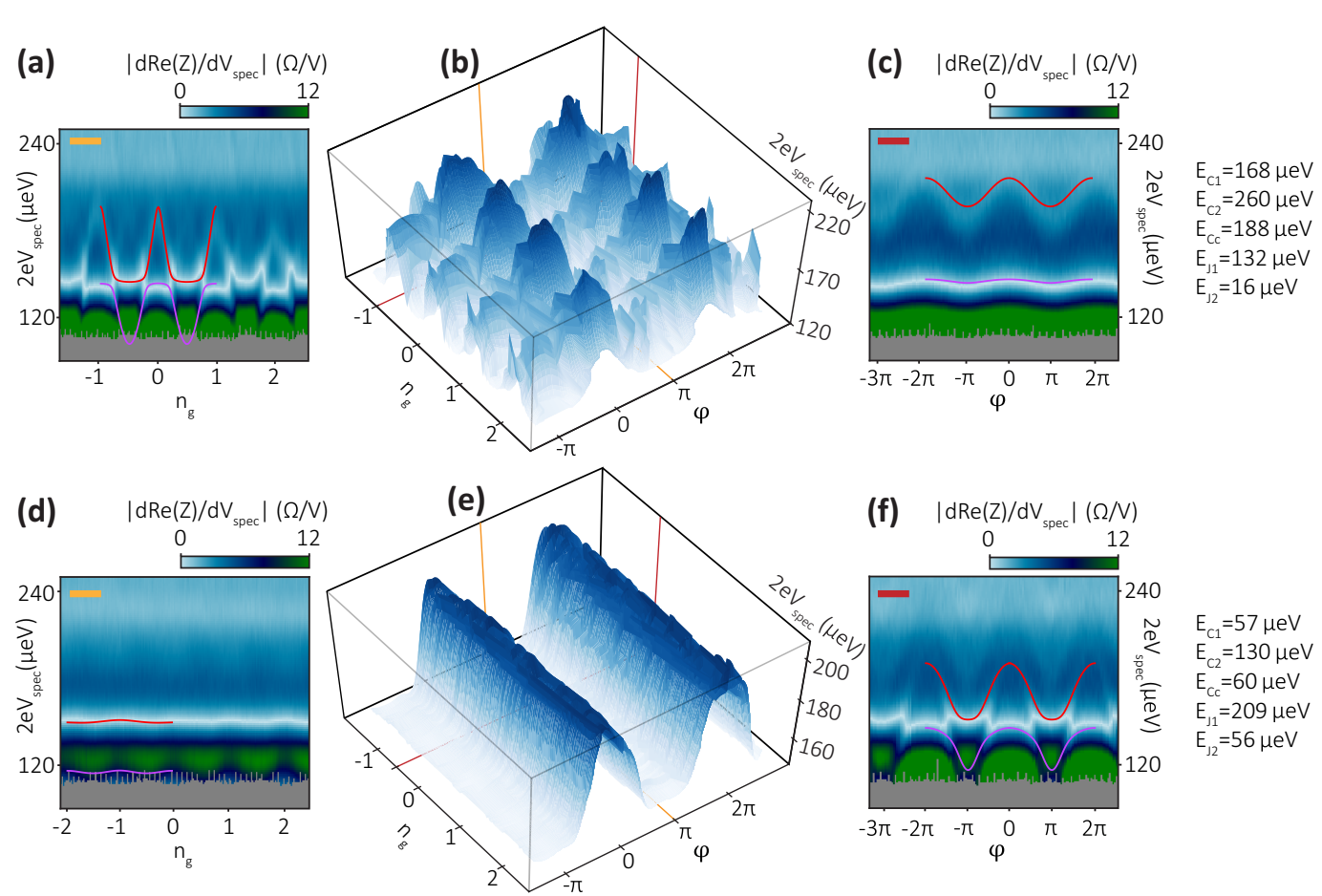

FIG. 3. Upper row: The measured excitations spectrum of device 1 as a function of $n_{\mathrm{g}}$ and $\varphi$ with $V_{\mathrm{tg} 1}=0.55 \mathrm{~V}, V_{\mathrm{tg} 2}=0.85 \mathrm{~V}$, and $V_{\mathrm{g}}=$ $250 \ldots 264.5 \mathrm{mV}$, same as in Fig. 2. (b) shows the full map of the second excitation whereas the line-cut data are shown at the positions denoted by the orange and red lines, respectively, in (a) and (c). Bottom row: Measured data on the same device with $V_{\mathrm{tg} 1}=1.5 \mathrm{~V}, V_{\mathrm{tg} 2}=1.545 \mathrm{~V}$, and $V_{\mathrm{g}}=916.9 \ldots 931.4 \mathrm{mV}$. Note the weak dependence on $n_{\mathrm{g}}$ due to the more open semiconductor channels. The best fits of the first two excitation energies are overlain in (a), (c) and (d), (e). All data were taken on device 1, and the parameters of the best fit are listed on the right for each setting. 
the charge dispersion is achieved by an $\approx 1 \mathrm{~V}$ increase in $V_{\mathrm{tg} 1}$ and $V_{\mathrm{tg} 2}$, showcasing the feasibility of topological quantum bit designs relying on the modulation of the charge dispersion in superconductor-semiconductor hybrid devices [26]. Furthermore, we observe a strong renormalization of the characteristic charging energies in the open regime [33,41], which does not exist for the case of fully metallic CPTs with tunnel junctions, where the charging energy is fully determined by the device geometry. In addition, we find an increase in the Josephson energies $E_{J 1,2}$, further contributing to the suppression of the charge dispersion of the CPT in the limit of $E_{J} \gg E_{C}$ [42].

Thus far, we only considered the even charge occupation of the island, where all electrons are part of the Cooper-pair condensate, and a single quasiparticle occupation is exponentially suppressed in $\Delta / k_{B} T$, where $\Delta$ is the superconducting gap [43]. However, a residual odd population is typically observed in the experiments, attributed to a nonthermal quasiparticle population in the superconducting circuit. In our experiment, we also find an additional spectral line, shifted by $\delta n_{\mathrm{g}}=0.5$ [see Figs. 2(b) and 3(a)], substantiating a finite odd number population of the island. We investigate this effect as a function of the temperature, and find that above a typical temperature of $T^{\star} \approx 300 \mathrm{mK}$, the measured signal is fully $1 e$ periodic [Fig. 4(b)], in contrast to the $2 e$ periodic data taken at $18 \mathrm{mK}$ [Fig. 4(a)].

To quantify the probability of the even and odd occupations, we extract the gate-charge-dependent component of the measured spectra $\delta I_{\text {spec }}\left(n_{\mathrm{g}}\right)$ to evaluate $\delta I_{\text {odd }}=\delta I_{\text {spec }}\left(n_{\mathrm{g}}=\right.$ $0.5)$ and $\delta I_{\text {even }}=\delta I_{\text {spec }}\left(n_{\mathrm{g}}=0\right)$ [see the inset in Fig. 4(c)]. We now make the assumption that the microwave photon frequency is much higher than the parity switching rate of the CPT. We evaluate the current response at $h f=2 e V_{\text {spec }}=$ $180 \mu \mathrm{eV}$ [see Figs. 4(a) and 4(b)] corresponding to $f=$ $43.5 \mathrm{GHz}$, well exceeding parity switching rates measured earlier on similar devices [31,44]. In this limit, the timeaveraged spectrometer response is the linear combination of the signals corresponding to the two parity states and $\delta I_{\text {even,odd }} \sim p_{\text {even,odd }}$, respectively. From this linear proportionality, $p_{\text {even }}=\left(1+\delta I_{\text {odd }} / \delta I_{\text {even }}\right)^{-1}$ follows.

We plot the extracted $p_{\text {even }}$ in Fig. 4(c). We find that above a crossover temperature $T^{\star} \approx 300 \mathrm{mK}, p_{\text {even }}$ approaches $1 / 2$, in agreement with the commonly observed breakdown of the parity effect at $T^{\star}<\Delta$ as a result of the vanishing even-odd free energy difference $[11,45,46]$,

$$
\Delta F=-k_{B} T \ln \tanh \left(N_{\mathrm{eff}} e^{-\Delta / k_{B} T}\right) .
$$

Here, $N_{\text {eff }}=\rho V \sqrt{2 \pi k_{B} T \Delta}$ at a temperature of $T$ with the island volume being $V$. We use the density of states at the Fermi level in the normal state $\rho=1.45 \times 10^{47} \mathrm{~J}^{-1} \mathrm{~m}^{-3}$ for aluminum [12]. Then the even charge parity occupation is given by $p_{\text {even }}=1-1 /\left(1+e^{\Delta F / k_{B} T}\right)$.

While this analysis describes the breakdown of the evenodd effect [see the blue dashed line as the best fit in Fig. 4(c)], it fails to account for the observed saturation $p_{\text {even }} \sim 0.8<1$ in the low-temperature limit, at $T<150 \mathrm{mK}$. This saturation can be be phenomenologically understood based on a spurious overheating of the island. We assume that the electron temperature $T_{e}=\left(T_{0}^{5}+T^{5}\right)^{1 / 5}$, where the chip (phonon)
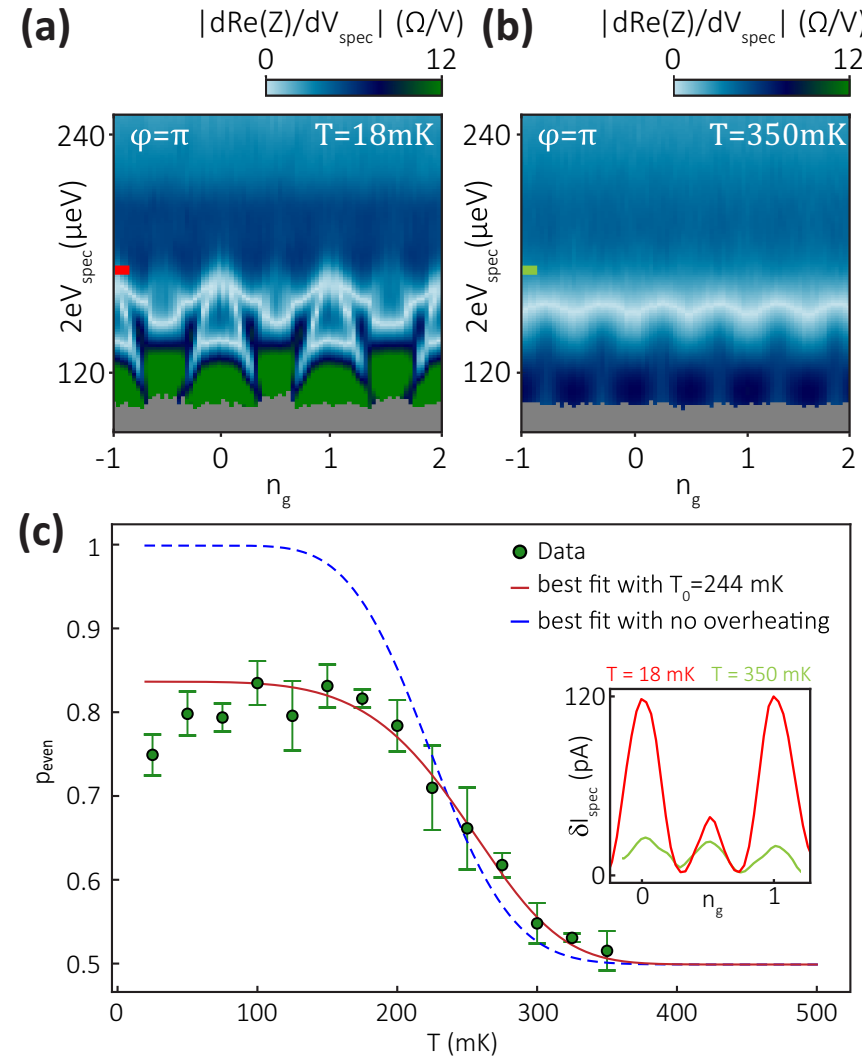

FIG. 4. The measured excitation spectra of device 2 at $V_{\mathrm{tg} 1}=$ $1.4 \mathrm{~V}, V_{\mathrm{tg} 2}=1.5025 \mathrm{~V}$ as a function of $n_{\mathrm{g}}$ corresponding to $V_{\mathrm{g}}=$ $-533 \ldots-527.8 \mathrm{mV}$ at $\varphi=\pi$ and at a temperature of $18 \mathrm{mK}$ (a) and $350 \mathrm{mK}$ (b). (c) The extracted even charge parity state occupation $p_{\text {even }}$ as a function of the temperature. The inset shows the modulation of the spectrometer current at $2 e V_{\text {spec }}=180 \mu \mathrm{eV}$ at these two temperatures, which defines $\delta I_{\text {odd }}$ and $\delta I_{\text {even }}$ (see text). The fit lines in (c) are based on Eq. (3), without (blue dashed line) and including overheating (solid red line).

temperature is $T$, and the electron saturation temperature is $T_{0}$ due to overheating and weak electron-phonon coupling at low temperatures [47].

The resulting best fit is shown as a solid red line in Fig. 4(c). We find a metallic volume of $V=4.66 \times 10^{-23} \mathrm{~m}^{3}$, consistent with the micrograph shown in Fig. 1(a). The fit yields a superconducting gap $\Delta=140 \pm 3 \mu \mathrm{eV}$, slightly lower than that of bulk aluminum, which is expected due to the presence of induced superconductivity in the semiconductor. The fitted saturation temperature $T_{0}=244 \pm$ $4 \mathrm{mK}$ and limiting $p_{\text {odd }}=1-p_{\text {even }} \approx 0.17$ demonstrates the abundance of nonequilibrium quasiparticles, in agreement with recent experimental findings $[48,49]$ on metallic devices. However, the unpaired quasiparticle density [46] $n_{\mathrm{qp}}=$ $V^{-1} N_{\text {eff }}^{2} \exp \left(-2 \Delta / k_{B} T_{0}\right) \approx 800 \mu \mathrm{m}^{-3}$ is orders of magnitude higher than typical values for all-metallic devices, falling in the range $n_{\mathrm{qp}} \approx 0.01 \ldots 1 \mu \mathrm{m}^{-3}[11,12,49]$. The same analysis was also performed on device 1 yielding similar results (see the Supplemental Material [34]). Our results substantiate the importance of controlling the quasiparticle population for hybrid semiconductor-superconductor CPTs in 
prospective topological quantum bits to decrease their rate of decoherence [50].

In conclusion, we performed broadband microwave spectroscopy on the gate charge and phase-dependent energy dispersion of InAs/Al hybrid CPTs, utilizing an on-chip nanofabricated circuit with a superconducting tunnel junction as a frequency-tunable microwave source. We understand the observed spectra based on the Hamiltonian of the circuit and find the characteristic charging and Josephson tunneling energy scales, both exhibiting strong modulation with the electrostatic gates coupled to the semiconductor channels. This broad tunability demonstrates the feasibility of prospective topological qubits relying on a controlled suppression of the charge modulation. Finally, we directly measure the time-averaged even and odd charge parity occupation of the CPT island, yielding a residual odd occupation probability and unpaired quasiparticle density which are much higher than typical values acquired earlier for all-metallic devices. This can be a limiting factor for topological quantum bit architectures that rely on charge parity manipulation and readout.

The analyzed raw data sets and data processing scripts for this publication are available at the 4TU.ResearchData repository [51].

The authors gratefully acknowledge O. Benningshof and R. Schouten for technical assistance as well as D. J. van Woerkom, D. Bouman, and B. Nijholt for fruitful discussions. This work was supported by the Netherlands Organization for Scientific Research (NWO) as part of the Frontiers of Nanoscience program, Microsoft Corporation Station Q, the Danish National Research Foundation, and a Synergy Grant of the European Research Council. P.K. acknowledges funding from the European Research Council (ERC) under Grant Agreement No. 716655(HEMs-DAM).
[1] D. V. Averin and K. K. Likharev, Single electronics: A correlated transfer of single electrons and Cooper pairs in systems of small tunnel junctions, in Mesoscopic Phenomena in Solids, edited by B. V. Alltshuler, P. A. Lee, and R. A. Webb (Elsevier, Amsterdam, 1991), p. 173.

[2] Single Charge Tunneling, edited by H. Grabert and M. H. Devoret (Plenum, New York, 1991).

[3] B. D. Josephson, Possible new effects in superconductive tunnelling, Phys. Lett. 1, 251 (1962).

[4] V. Ambegaokar and A. Baratoff, Tunneling Between Superconductors, Phys. Rev. Lett. 10, 486 (1963).

[5] T. A. Fulton and G. J. Dolan, Observation of Single-Electron Charging Effects in Small Tunnel Junctions, Phys. Rev. Lett. 59, 109 (1987).

[6] L. J. Geerligs, V. F. Anderegg, J. Romijn, and J. E. Mooij, Single Cooper-Pair Tunneling in Small-Capacitance Junctions, Phys. Rev. Lett. 65, 377 (1990).

[7] M. T. Tuominen, J. M. Hergenrother, T. S. Tighe, and M. Tinkham, Experimental Evidence for Parity-Based $2 e$ Periodicity in a Superconducting Single-Electron Tunneling Transistor, Phys. Rev. Lett. 69, 1997 (1992).

[8] P. Joyez, P. Lafarge, A. Filipe, D. Esteve, and M. H. Devoret, Observation of Parity-Induced Suppression of Josephson Tunneling in the Superconducting Single Electron Transistor, Phys. Rev. Lett. 72, 2458 (1994).

[9] T. M. Eiles and J. M. Martinis, Combined Josephson and charging behavior of the supercurrent in the superconducting single-electron transistor, Phys. Rev. B 50, 627 (1994).

[10] J. Aumentado, M. W. Keller, J. M. Martinis, and M. H. Devoret, Nonequilibrium Quasiparticles and $2 e$ Periodicity in SingleCooper-Pair Transistors, Phys. Rev. Lett. 92, 066802 (2004).

[11] D. J. van Woerkom, A. Geresdi, and L. P. Kouwenhoven, One minute parity lifetime of a NbTiN Cooper-pair transistor, Nat. Phys. 11, 547 (2015).

[12] A. J. Ferguson, N. A. Court, F. E. Hudson, and R. G. Clark, Microsecond Resolution of Quasiparticle Tunneling in the SingleCooper-Pair Transistor, Phys. Rev. Lett. 97, 106603 (2006).

[13] M. D. Shaw, R. M. Lutchyn, P. Delsing, and P. M. Echternach, Kinetics of nonequilibrium quasiparticle tunneling in superconducting charge qubits, Phys. Rev. B 78, 024503 (2008).
[14] P.-M. Billangeon, F. Pierre, H. Bouchiat, and R. Deblock, Very High Frequency Spectroscopy and Tuning of a Single-CooperPair Transistor with an On-Chip Generator, Phys. Rev. Lett. 98, 126802 (2007).

[15] P.-M. Billangeon, F. Pierre, H. Bouchiat, and R. Deblock, ac Josephson Effect and Resonant Cooper Pair Tunneling Emission of a Single Cooper Pair Transistor, Phys. Rev. Lett. 98, 216802 (2007).

[16] P. Krogstrup, N. L. B. Ziino, W. Chang, S. M. Albrecht, M. H. Madsen, E. Johnson, J. Nygård, C. M. Marcus, and T. S. Jespersen, Epitaxy of semiconductor-superconductor nanowires, Nat. Mater. 14, 400 (2015).

[17] S. Gazibegovic, D. Car, H. Zhang, S. C. Balk, J. A. Logan, M. W. A. de Moor, M. C. Cassidy, R. Schmits, D. Xu, G. Wang, P. Krogstrup, R. L. M. Op het Veld, K. Zuo, Y. Vos, J. Shen, D. Bouman, B. Shojaei, D. Pennachio, J. S. Lee, P. J. van Veldhoven et al., Epitaxy of advanced nanowire quantum devices, Nature (London) 548, 434 (2017).

[18] D. J. van Woerkom, A. Proutski, B. van Heck, D. Bouman, J. I. Väyrynen, L. I. Glazman, P. Krogstrup, J. Nygård, L. P. Kouwenhoven, and A. Geresdi, Microwave spectroscopy of spinful Andreev bound states in ballistic semiconductor Josephson junctions, Nat. Phys. 13, 876 (2017).

[19] M. Hays, G. de Lange, K. Serniak, D. J. van Woerkom, D. Bouman, P. Krogstrup, J. Nygård, A. Geresdi, and M. H. Devoret, Direct Microwave Measurement of Andreev-BoundState Dynamics in a Semiconductor-Nanowire Josephson Junction, Phys. Rev. Lett. 121, 047001 (2018).

[20] L. Tosi, C. Metzger, M. F. Goffman, C. Urbina, H. Pothier, S. Park, A. L. Yeyati, J. Nygård, and P. Krogstrup, Spin-Orbit Splitting of Andreev States Revealed by Microwave Spectroscopy, Phys. Rev. X 9, 011010 (2019).

[21] T. W. Larsen, K. D. Petersson, F. Kuemmeth, T. S. Jespersen, P. Krogstrup, J. Nygård, and C. M. Marcus, SemiconductorNanowire-Based Superconducting Qubit, Phys. Rev. Lett. 115, 127001 (2015).

[22] G. de Lange, B. van Heck, A. Bruno, D. J. van Woerkom, A. Geresdi, S. R. Plissard, E. P. A. M. Bakkers, A. R. Akhmerov, and L. DiCarlo, Realization of Microwave Quantum Circuits Using Hybrid Superconducting-Semiconducting 
Nanowire Josephson Elements, Phys. Rev. Lett. 115, 127002 (2015).

[23] F. Luthi, T. Stavenga, O. W. Enzing, A. Bruno, C. Dickel, N. K. Langford, M. A. Rol, T. S. Jespersen, J. Nygård, P. Krogstrup, and L. DiCarlo, Evolution of Nanowire Transmon Qubits and Their Coherence in a Magnetic Field, Phys. Rev. Lett. 120, 100502 (2018).

[24] L. Casparis, N. J. Pearson, A. Kringhøj, T. W. Larsen, F. Kuemmeth, J. Nygård, P. Krogstrup, K. D. Petersson, and C. M. Marcus, Voltage-controlled superconducting quantum bus, Phys. Rev. B 99, 085434 (2019).

[25] T. Hyart, B. van Heck, I. C. Fulga, M. Burrello, A. R. Akhmerov, and C. W. J. Beenakker, Flux-controlled quantum computation with Majorana fermions, Phys. Rev. B 88, 035121 (2013).

[26] D. Aasen, M. Hell, R. V. Mishmash, A. Higginbotham, J. Danon, M. Leijnse, T. S. Jespersen, J. A. Folk, C. M. Marcus, K. Flensberg, and J. Alicea, Milestones Toward Majorana-Based Quantum Computing, Phys. Rev. X 6, 031016 (2016).

[27] T. Karzig, C. Knapp, R. M. Lutchyn, P. Bonderson, M. B. Hastings, C. Nayak, J. Alicea, K. Flensberg, S. Plugge, Y. Oreg, C. M. Marcus, and M. H. Freedman, Scalable designs for quasiparticle-poisoning-protected topological quantum computation with Majorana zero modes, Phys. Rev. B 95, 235305 (2017).

[28] S. Plugge, A. Rasmussen, R. Egger, and K. Flensberg, Majorana box qubits, New J. Phys. 19, 012001 (2017).

[29] S. M. Albrecht, A. P. Higginbotham, M. Madsen, F. Kuemmeth, T. S. Jespersen, J. Nygård, P. Krogstrup, and C. M. Marcus, Exponential protection of zero modes in Majorana islands, Nature (London) 531, 206 (2016).

[30] J. Shen, S. Heedt, F. Borsoi, B. van Heck, S. Gazibegovic, R. L. M. Op het Veld, D. Car, J. A. Logan, M. Pendharkar, S. J. J. Ramakers, G. Wang, D. Xu, D. Bouman, A. Geresdi, C. J. Palmstrm, E. P. A. M. Bakkers, and L. P. Kouwenhoven, Parity transitions in the superconducting ground state of hybrid InSb-Al Coulomb islands, Nat. Commun. 9, 4801 (2018).

[31] J. van Veen, A. Proutski, T. Karzig, D. I. Pikulin, R. M. Lutchyn, J. Nygård, P. Krogstrup, A. Geresdi, L. P. Kouwenhoven, and J. D. Watson, Magnetic-field-dependent quasiparticle dynamics of nanowire single-Cooper-pair transistors, Phys. Rev. B 98, 174502 (2018).

[32] K. Zuo, V. Mourik, D. B. Szombati, B. Nijholt, D. J. van Woerkom, A. Geresdi, J. Chen, V. P. Ostroukh, A. R. Akhmerov, S. R. Plissard, D. Car, E. P. A. M. Bakkers, D. I. Pikulin, L. P. Kouwenhoven, and S. M. Frolov, Supercurrent Interference in Few-Mode Nanowire Josephson Junctions, Phys. Rev. Lett. 119, 187704 (2017).

[33] D. V. Averin, Coulomb Blockade in Superconducting Quantum Point Contacts, Phys. Rev. Lett. 82, 3685 (1999).

[34] See Supplemental Material at http://link.aps.org/supplemental/ 10.1103/PhysRevB.99.220504 for details on fabrication, device modeling, and for additional data sets.

[35] L. Bretheau, Ç. Ö. Girit, H. Pothier, D. Esteve, and C. Urbina, Exciting Andreev pairs in a superconducting atomic contact, Nature (London) 499, 312 (2013).

[36] T. Holst, D. Esteve, C. Urbina, and M. H. Devoret, Effect of a Transmission Line Resonator on a Small Capacitance Tunnel Junction, Phys. Rev. Lett. 73, 3455 (1994).
[37] F. Kos, S. E. Nigg, and LI Glazman, Frequency-dependent admittance of a short superconducting weak link, Phys. Rev. B 87, 174521 (2013).

[38] A. Blais, R.-Sh. Huang, A. Wallraff, S. M. Girvin, and R. J. Schoelkopf, Cavity quantum electrodynamics for superconducting electrical circuits: An architecture for quantum computation, Phys. Rev. A 69, 062320 (2004).

[39] U. Vool and M. Devoret, Introduction to quantum electromagnetic circuits, Int. J. Circuit Theory Appl. 45, 897 (2017).

[40] A. Wallraff, D. I. Schuster, A. Blais, L. Frunzio, R.-S. Huang, J. Majer, S. Kumar, S. M. Girvin, and R. J. Schoelkopf, Strong coupling of a single photon to a superconducting qubit using circuit quantum electrodynamics, Nature (London) 431, 162 (2004).

[41] D. Pikulin, K. Flensberg, L. I. Glazman, M. Houzet, and R. M. Lutchyn, Coulomb Blockade of a Nearly Open Majorana Island, Phys. Rev. Lett. 122, 016801 (2019).

[42] J. Koch, T. M. Yu, J. Gambetta, A. A. Houck, D. I. Schuster, J. Majer, A. Blais, M. H. Devoret, S. M. Girvin, and R. J. Schoelkopf, Charge-insensitive qubit design derived from the Cooper pair box, Phys. Rev. A 76, 042319 (2007).

[43] D. V. Averin and Yu. V. Nazarov, Single-Electron Charging of a Superconducting Island, Phys. Rev. Lett. 69, 1993 (1992).

[44] S. M. Albrecht, E. B. Hansen, A. P. Higginbotham, F. Kuemmeth, T. S. Jespersen, J. Nygård, P. Krogstrup, J. Danon, K. Flensberg, and C. M. Marcus, Transport Signatures of Quasiparticle Poisoning in a Majorana Island, Phys. Rev. Lett. 118, 137701 (2017).

[45] P. Lafarge, P. Joyez, D. Esteve, C. Urbina, and M. H. Devoret, Measurement of the Even-Odd Free-Energy Difference of an Isolated Superconductor, Phys. Rev. Lett. 70, 994 (1993).

[46] A. P. Higginbotham, S. M. Albrecht, G. Kiršanskas, W. Chang, F. Kuemmeth, P. Krogstrup, T. S. Jespersen, J. Nygård, K. Flensberg, and C. M. Marcus, Parity lifetime of bound states in a proximitized semiconductor nanowire, Nat. Phys. 11, 1017 (2015).

[47] F. Giazotto, T. T. Heikkilä, A. Luukanen, A. M. Savin, and J. P. Pekola, Opportunities for mesoscopics in thermometry and refrigeration: Physics and applications, Rev. Mod. Phys. 78, 217 (2006).

[48] K. Serniak, M. Hays, G. de Lange, S. Diamond, S. Shankar, L. D. Burkhart, L. Frunzio, M. Houzet, and M. H. Devoret, Hot Nonequilibrium Quasiparticles in Transmon Qubits, Phys. Rev. Lett. 121, 157701 (2018).

[49] E. T. Mannila, V. F. Maisi, H. Q. Nguyen, C. M. Marcus, and J. P. Pekola, Parity effect does not mean a superconductor free of quasiparticles, arXiv:1807.01733.

[50] D. Rainis and D. Loss, Majorana qubit decoherence by quasiparticle poisoning, Phys. Rev. B 85, 174533 (2012).

[51] A. Proutski, D. Laroche, B. van 't Hooft, P. Krogstrup, J. Nygard, L. P. Kouwenhoven, and A. Geresdi, Broadband microwave spectroscopy of semiconductor nanowire-based Cooper-pair transistors, 4TU.ResearchData repository, http://dx.doi.org/10.4121/uuid:5d54f11b-6774-4ae4-96cf25e6a91927e2. 